\title{
ON-LINE TEXTURE MEASUREMENTS FOR THE PREDICTION OF THE ANISOTROPY OF MAGNETIC PROPERTIES
}

\author{
P. BLANDFORD and J. A. SZPUNAR \\ Department of Mining and Metallurgical Engineering, McGill University, Canada
}

An outline of a system to be used for on-line determination of texture is presented in this paper; information is given as to the detection geometry and equipment as well as the method whereby the orientation distribution function (ODF) will be calculated. Based on coefficients claculated in a simulation of the on-line system, a number of correlations, including relative permeability, hysteresis losses, magnetic torque and others, are carried out.

KEY WORDS X-ray diffraction, position sensitive detector, back reflection method, texture coefficients, magnetic anisotropy.

The benefits of an on-line texture measurement system are immediately evident in the ongoing search for better quality and processing control. Information collected directly on the production line can be used to correct the tecinological parameters used in the product's manufacture in order to obtain superior quality, or to ensure that a set standard of product is maintained. The method most readily applicable to the measurement of texture is X-ray diffraction; either by energy or angular dispersive methods, these methods have been proven and provide a high degree of versatility in their capabilities to correlate with and measure material properties. At present, there is an on-line system working in Karlsruhe, Germany ${ }^{1}$ which is used to measure selected texture maxima in order to predict the plastic strain ratio.

It is the intent of the authors to build a texture measuring system (using a position sensitive detector (PSD)) applied to on-line determination of the crystallite orientation distribution function. Such fully quantitative information will then be used to predict the anisotropy of magnetic properties. In this paper we present the first methodological results concerning the measurement and calculation of the ODF in the on-line geometry and also the work done to correlate magnetic properties and texture.

\section{DESCRIPTION OF ON-LINE SYSTEM FOR TEXTURE MEASUREMENTS}

Our system uses a standard $50 \mathrm{kV}$ X-ray generator and a curved $120^{\circ} \mathrm{PSD}$ in the geometry shown in Figure 1. The sheet is rolled across in front of the beam providing a high degree of statistical averaging in the accumulation of data. Both 


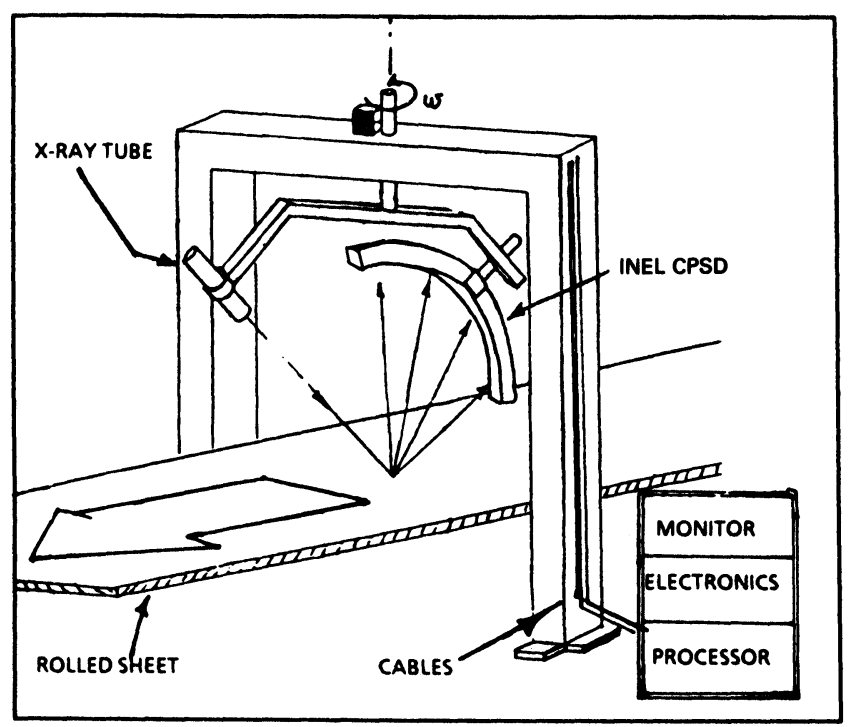

Figure 1 Detection geometry used for on-line texture data collection.

the X-ray tube and detector are fixed relative to each other by an arm that is used to rotate the projection of the incident beam on the sheet from 0 to 90 degrees from the rolling direction; the plane containing the curvature of the detector will generally be inclined at about 30 degrees from the sheet normal. Both the stepper motors used to accomplish the rotations and the detector electronics are controlled by communication with a computerized work station set up on the plant floor. Utilizing the detector in this geometry will allow us to determine pole densities along certain lines in up to 14 different pole figures simultaneously. This detection system and geometry has been chosen in order to optimize the total number of useful pole density data obtained, and to maximize the range of applications for which the system can be used.

Ordinarily, because of the large grain size $(10 \mathrm{~mm})$ of oriented $\mathrm{Fe}-\mathrm{Si}$ steel, neutron diffraction is one of the few ways by which pole density data can be obtained for ODF calculation; however, because in the stated geometry the specimen will always be moving past the incident beam, statistically reliable results will always be obtained.

The building of the system will proceed in two stages; the first, which is presently being accomplished, is to build a laboratory system which collects data in the same way as the design but does so without the required scale of building. The building of the actual design prototype will proceed after full testing has been carried out on the laboratory model.

\section{ON-LINE DETERMINATION OF THE ODF}

The orientation of crystallites is described by a distribution function of the three Euler angles. This function is ordinarily determined from three complete 
experimental pole figures, which allows the orthogonality relations of the surface spherical harmonics to be used in the calculation. The ODF can also be determined from the more easily obtained reflection part of the pole figures, although this entails more laborious calculations. Our on-line texture measurements will allow the determination of the pole density values in a number of pole figures simultaneously. The number of points on each pole figure, however, is greatly reduced and thus a method of calculation must be used which does not rely on the integration of densities over the entire space of the pole figure. The mathematical principle of such methods has been suggested by Bunge. ${ }^{2}$ Using this technique, a fit between the theoretical ODF and arbitrary pole figure or inverse pole figure data points is postulated. The calculation of the ODF coefficients requires the minimization of the following expression.

$$
\sum_{i} \sum_{j}\left[p_{i j} N_{j}-P_{j}^{t h}\right]^{2} W_{i j}=\text { minimum }
$$

where $P_{i j}$ and $P_{j}^{\text {th }}$ are the pole density and theoretical pole density measured on pole figure $j, N_{j}$ is a normalization factor used to reduce the density values to units corresponding to times random units, and $W_{i j}$ is a weighting factor.

In order to simulate on-line texture measurements using the detection geometry described above, without directly measuring the pole densities, pole figures were calculated using select pole figure data taken from a series of calculated complete pole figures. These complete pole figures were obtained from the ODF series expansion coefficients calculated using three full pole figures measured by neutron diffraction in a highly Goss oriented $\mathrm{Fe}-\mathrm{Si}$ steel specimen.

Data obtained in this way consists of a series of pole densities along different lines of the different pole figures. Because such limited number of data will not allow us to determine the coefficients of the ODF as well as the normalization constants $N_{j}$, (Eq. (1)) it is necessary to normalize the data first.

Table 1 compares the coefficients of the ODF up to $l=8$ calculated using data simulating those which would be collected from the on-line measurement geometry to the coefficients of the ODF used to calculate the complete pole figure data.

Table 1 Comparison of coefficients of the ODF calculated by program to simulate on-line detection system to the original data.

\begin{tabular}{lrr}
\hline Coefficients & Calculated & Original \\
\hline$C_{4}^{11}$ & 0.014 & 0.008 \\
$C_{4}^{12}$ & -5.166 & -6.186 \\
$C_{4}^{13}$ & 2.869 & 3.612 \\
$C_{6}^{11}$ & -4.402 & -4.171 \\
$C_{6}^{12}$ & 1.562 & 2.055 \\
$C_{6}^{13}$ & 2.031 & 2.604 \\
$C_{6}^{14}$ & 2.770 & 3.663 \\
$C_{8}^{11}$ & 2.127 & 2.294 \\
$C_{8}^{12}$ & -0.464 & -0.627 \\
$C_{8}^{13}$ & 3.714 & 4.371 \\
$C_{8}^{14}$ & -3.835 & -4.648 \\
$C_{8}^{15}$ & 2.581 & 2.450 \\
\hline
\end{tabular}




\section{CORRELATING THE ANISOTROPY OF MAGNETIC PROPERTIES WITH TEXTURE}

The anisotropy of the properties of many single crystals can be represented, in the reference frame of the crystal, by a polynomial series expansion of the form

$$
P(\bar{h})=A_{0}^{\prime}+A_{4}^{\prime} \Phi_{4}^{\prime}(\bar{h})+A_{6}^{\prime} \Phi_{6}^{\prime}(\bar{h})+\ldots
$$

where the basis functions $\Phi_{i}^{\prime}$ are invariant with respect to the relevant crystal symmetry operations. Assuming then, that the anisotropy of bulk properties of polycrystalline specimens are just the simple average of the constituent single crystal anisotropies, ${ }^{3}$ an analogous expression can be written in a manner similar to that done by Morris and Flowers ${ }^{4}$ for some directionally dependant properties of polycrystalline specimens, i.e.

$$
F(\alpha, \beta)=A_{0}+A_{4} \Phi_{4}(\alpha, \beta)+A_{6} \Phi_{6}(\alpha, \beta)+\ldots
$$

where $\Phi_{i}(\alpha, \beta)$ are the basis functions used in Eq. (2) that have been suitably averaged through the ODF of the specimen, and where $\alpha$ and $\beta$ are the spherical angular coordinates of the direction in which the property is to be measured with respect to the coordinate system fixed within the specimen.

Following Bunge, ${ }^{5}$ who derived a similar expression specifically for the averaged anisotropy of magnetization energy, one can write $\Phi_{4}(\alpha, \beta)$ and $\Phi_{6}(\alpha, \beta)$ of Eq. (3) as

$$
\begin{gathered}
\Phi_{4}(\alpha, \beta)=\frac{1}{9 n_{4} \sqrt{ } \pi} \frac{1}{5} F_{4}(\alpha, \beta) \\
\Phi_{6}(\alpha, \beta)=\frac{\Phi_{4}(\alpha, \beta)}{11}+\frac{1}{13 n_{6} \sqrt{ } \pi} \frac{1}{231} F_{6}(\alpha, \beta)
\end{gathered}
$$

where

$$
\begin{aligned}
F_{4}(\alpha, \beta)= & \frac{1}{\sqrt{ } 2} C_{4}^{11} \bar{P}_{4}^{0}(\cos \alpha)+C_{4}^{12} \bar{P}_{4}^{2}(\cos \alpha) \cos ^{2} \beta+C_{4}^{13} \bar{P}_{4}^{4}(\cos \alpha) \cos ^{4} \beta \\
F_{6}(\alpha, \beta)= & \frac{1}{\sqrt{ } 2} C_{6}^{11} \bar{P}_{6}^{0}(\cos \alpha)+C_{6}^{12} \bar{P}_{6}^{2}(\cos \alpha) \cos ^{2} \beta+C_{6}^{13} \bar{P}_{6}^{4}(\cos \alpha) \cos ^{4} \beta \\
& +C_{6}^{14} \bar{P}_{6}^{4}(\cos \alpha) \cos ^{6} \beta
\end{aligned}
$$

For details on the exact manner in which Eq. (4) was derived, refer to Bunge ${ }^{5}$ or to a review article by Szpunar. ${ }^{6}$

\section{CORRELATING MAGNETOCRYSTALLINE ENERGY AND TORQUE WITH TEXTURE}

Magnetic torque is a measure of the derivative of the magnetocrystalline energy (see Eq. (5)) and can be used to estimate $A_{4}$, and $A_{6}$, which, according to [7] and [4], are related to the single crystal anisotropy constants $K_{4}$, and $K_{6}$.

$$
\tau(\beta)=-\frac{d E(\beta)}{d \beta} ; \quad \alpha=\pi / 2
$$




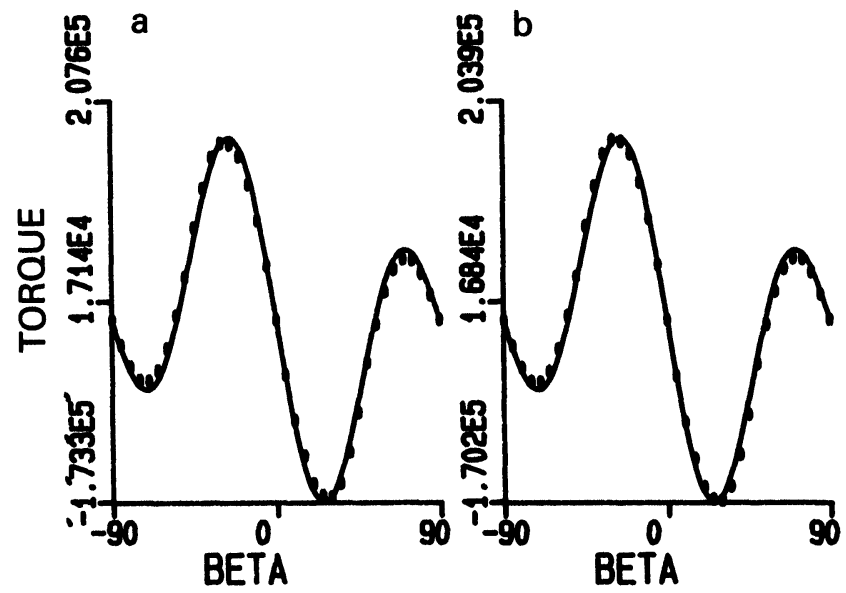

Figure 2 Magnetic torque vs $\beta$ fitted using a) $\Phi_{4}$ and b) $\Phi_{4}+\Phi_{6}$. Legend: - least square fit, $\bigcirc$ data points.

As an illustration, torque data obtained from measurements taken on a highly oriented $\mathrm{Fe}-\mathrm{Si}$ steel sample was fitted with variations of the torque equation, the results of which are presented in Figure 2.

The two fits differ from one another in that Figure $2 \mathrm{a}$ uses a correlation assuming that $A_{6}=0$, and Figure $2 \mathrm{~b}$ does not. It is evident that, within the errors typical in these measurements, their is no significant improvement acquired by adding the higher order $\Phi_{6}$ term.

\section{CORRELATING THE PROPERTIES OF MAGNETIZATION CURVES WITH TEXTURE}

The understanding of the different processes through which the domain structures pass upon magnetization is understood in terms of theories that minimize the various energies involved. In the simpler cases of single crystals or ideally random polycrystalline samples, the processes of domain wall bowing, domain wall motion and domain nucleation in directions closer to that of the applied field, and then domain rotation are usually distinct enough to be separable on the magnetization curves. However, because of the complexity of the associated magnetization curves in oriented $\mathrm{Fe}-\mathrm{Si}$ steels, such distinction is difficult. Therefore, for analysis of the magnetization changes we will use an approach based on the thermodynamics of the changes in the domain structure, Brady ${ }^{8}$ suggests that the magnetization curves can be divided into separate processes by plotting the logarithm of the specimens' magnetization against the logarithm field strength applied to it; the plots split into a sequence of intersecting lines, each of which can be modeled with an equation of the form

$$
\log M=k_{i} \log H_{a}+c_{i}
$$

where $H_{a}$ and $M$ are the applied magnetic field strength and specimen magnetization, respectively, and $k_{i}$ and $c_{i}$ are fitting constants applicable to the 

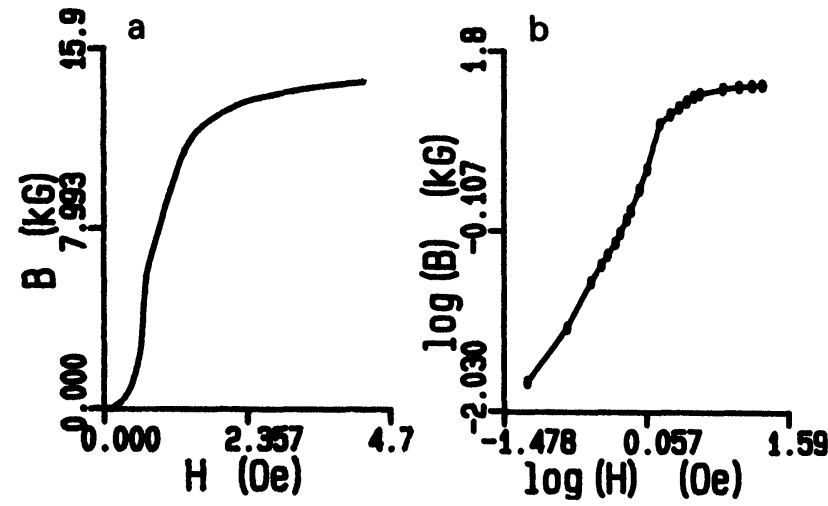

Figure 3 Magnetization curves a) $B(\mathrm{kG})$ vs $H_{a}(\mathrm{Oe})$ and b) $B(\mathrm{kG})$ vs $\log H_{a}$ obtained along direction perpendicular to the rolling.

curves in the $i$ th thermodynamic regime. A linear relationship between $\log H_{a}$ and $\log M$ means that $M$ is proportional to the $k_{i}$ power of $H_{a}$ in the following way.

$$
M=10^{c_{i}} H_{a}^{k_{i}}
$$

This means that, provided that the relative permeability is greater than 100

to an accuracy of $1 \%$.

$$
B=10^{c_{i}} H_{a}^{k_{i}}
$$

$\log B$ vs $\log H_{a}$ plots created from the magnetization curves of 10 Epstein strips (every 10 degrees from rolling direction to transverse direction) were used to create the following series of plots and values. Figure 3 contains typical magnetization curves and associated $\log -\log$ plots. Table 2 contains the position of the second and third transition points and the permeabilities measured at these points.

Contrary to the maximum five which Brady's model suggests, there are possibly up to seven different processes constituting the magnetization curves. The regions

Table 2 Relative permeability, $\mu_{r}$ for all Epstein strips measured at applied field strengths corresponding to the transition points.

\begin{tabular}{rllll}
\hline $\boldsymbol{\beta}\left(^{\circ}\right)$ & $\begin{array}{l}\text { Transition } 2 \\
H(O e)\end{array}$ & $\begin{array}{l}\text { Transition } 2 \\
\mu_{r} \pm 5 \%\end{array}$ & $\begin{array}{l}\text { Transition } 3 \\
H(O e)\end{array}$ & $\begin{array}{l}\text { Transition } 3 \\
\mu_{r} \pm 5 \%\end{array}$ \\
\hline 0 & 0.068 & 23,500 & 0.106 & 82,800 \\
10 & 0.072 & 13,900 & 0.200 & 46,200 \\
20 & 0.1 & 6400 & 0.299 & 24,700 \\
30 & 0.115 & 3480 & 0.367 & 18,200 \\
40 & 0.162 & 2720 & 0.475 & 12,000 \\
50 & 0.193 & 1760 & 0.575 & 8170 \\
60 & 0.245 & 1020 & n/a & - \\
70 & 0.231 & 1260 & n/a & - \\
80 & 0.266 & 2440 & 0.556 & 11,700 \\
90 & 0.316 & 2860 & 0.507 & 10,800 \\
\hline
\end{tabular}


are entirely distinct and one can follow most of them through the sequence of the Epstein strips, thus quantifying the anisotropy existing in the Fe-Si steel. Without necessarily understanding the exact process whereby the strips' magnetization changes in each of these regions, it is possible to theoretically understand in what manner the anisotropy of bulk properties such as the relative permeability, susceptibility or even the magnitude of $H_{a}$ as measured at the transition points shown in Figure 3, correlates with texture.

For example, the anisotropy of permeability in the single crystal is very distinct in iron and thus, provided that there are no other anisotropies derived from sources other than that due to single crystal anisotropies, then functions of the form of Eq. (3) are suited for correlation. This statement is generally true whether the permeability values are taken at a specific value of $H_{a}$ or at a characteristic point on the magnetization curve, such as the aforementioned transition points. However, even though the value of $H_{a}$ at the transitions on the $\log -\log$ curves of single crystal iron exhibit an anisotropy, there is some doubt in using functions such as Eq. (3) to fit the anisotropy of position of these knees in the polycrystalline specimen, because, according to Sizoo's data, ${ }^{9}$ the single
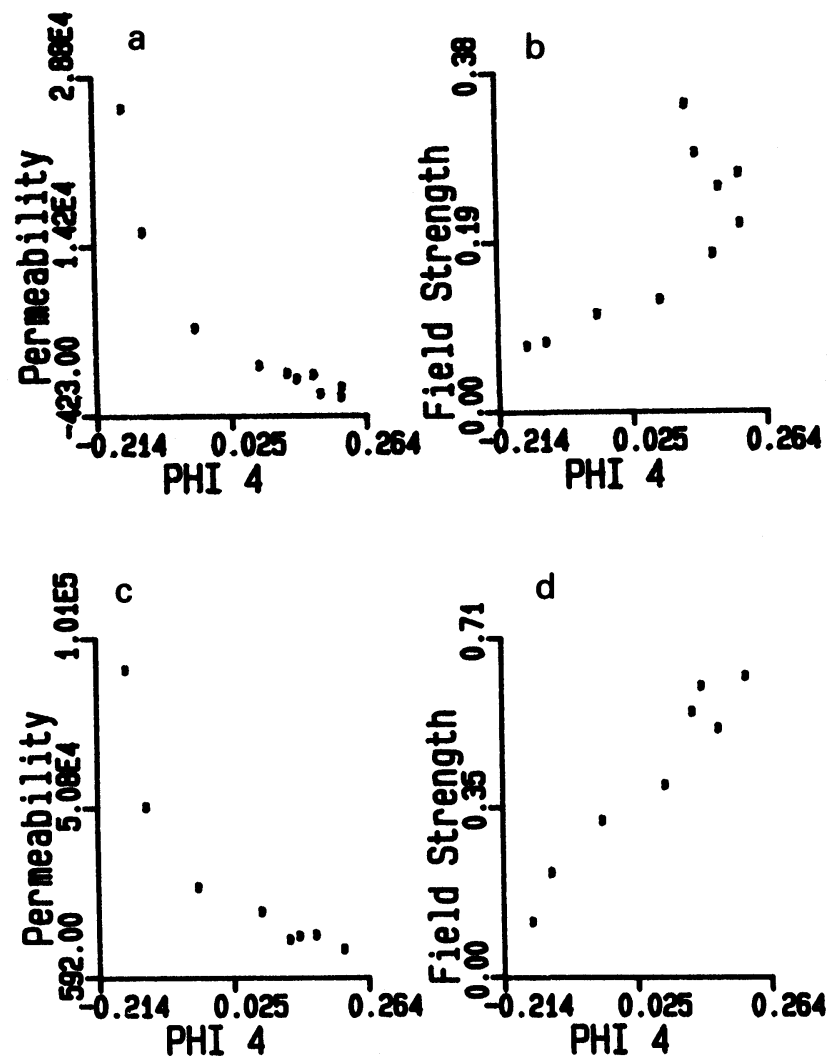

Figure 4 Curves represent: a) relative permeability at transition 2 vs $\Phi_{4}$, b) field strength at transition 2 vs $\Phi_{4}$, c) relative permeability at transition 3 vs $\Phi_{4}$, d) field strength at transition 3 vs $\Phi_{4}$, assuming $A_{6}=0$. 
crystal exhibits only two transitions; although there are no guarantees that Sizoo's data represent the general case, this increases the possibility that there are other transitions existing in the curves of the polycrystalline specimen which are caused by factors other than single crystal anisotropies.

Figure 4 are the results of a number of tests using Eq. (3) to correlate the permeability and applied magnetic field strengths as measured at the transitions
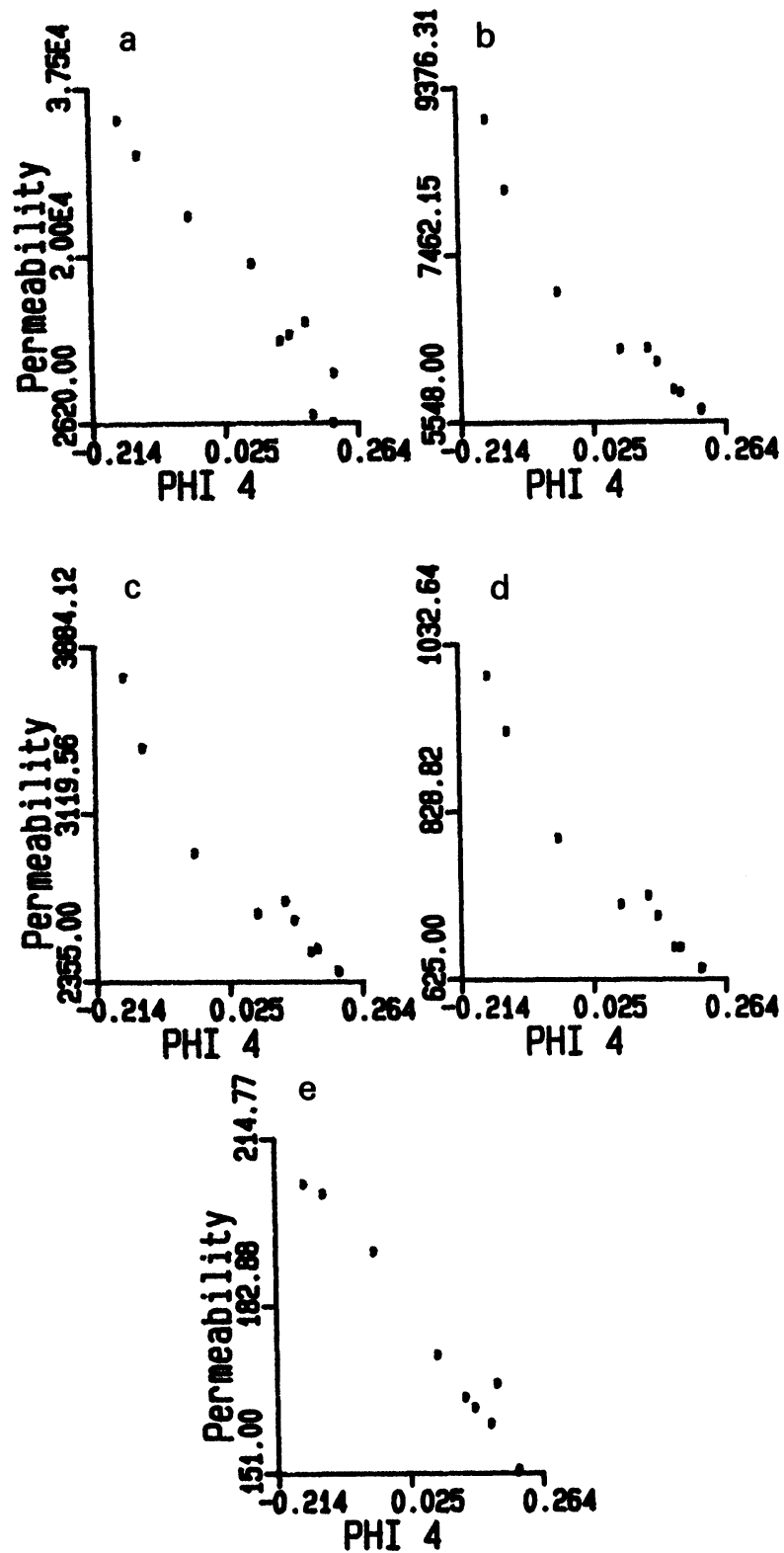

Figure 5 Relative permeability versus $\Phi_{4}$ measured at a) $0.5 \mathrm{Oe}$, b) $2.0 \mathrm{Oe}$, c) $5.0 \mathrm{Oe}$, d) $20.0 \mathrm{Oe}$, and e) 100.0 Oe with Eq. (3), assuming $A_{6}=0$. 




Figure 6 Relative permeability vs $\Phi_{4}$ measured at a) $0.1 \mathrm{kG}, \mathrm{b}$ ) $0.2 \mathrm{kG}$ (Eq. (3), assuming $A_{6}=0$ ).

joining the various regions on the log-log plots. The results depict relatively good correlation of the texture with the relative permeability for all samples but the 0 and $10^{\circ}$ Epstein strips; however, there is mixed agreement between the tested correlations with the field data. Similar correlations of the field data with $\Phi_{4}$ for the fourth and fifth transition points provide as good results as those shown for the third transition point data.

In order to complete this section, a variety of plots are presented showing the flexibility in correlating the relative permeability with texture through Eq. (3); the plots in Figure 5 and Figure 6 describe the degree to which permeabilities measured at applied field values of $0.5,2.0,5.0,20.0$, and $100.0 \mathrm{Oe}$ and initial relative permeability measured at 0.1 and $0.2 \mathrm{kG}$ correlate with texture.

In general, the quality of correlation between texture and the anisotropy of the permeability shown in the Figures 5 and 6 increases with increasing $H$, the major problems being that the data measured for $\beta=0$ and $10^{\circ}$ are high with respect to the other data and that the data for $\beta>60^{\circ}$ are sometimes too low. It was found that the quality of fit did not improve sufficiently to warrant correlating with the higher order terms existing in the texture function so all plots assume that $A_{6}=0$.

\section{CORRELATING HYSTERESIS LOSSES WITH TEXTURE}

The irreversible processes leading to the hysteresis losses are associated with the rearrangement of domain structure which are chiefly affected by metallurgical variables such as grain size, orientation, and purity, and other factors such as the state of internal stress and the interaction between the main and supplementary domain structure. The rearrangement of these structures usually involves a combination of domain wall motion, domain wall bowing and domain wall nucleation, each of which can occur in various ratios. Figure 7 shows the manner in which the anisotropy of hysteresis losses of $\mathrm{Fe}-\mathrm{Si}$ steel correlate with the texture function, assuming that $A_{6}=0$.

Phenomenologically, it should be possible to correlate the anisotropy of hysteresis losses with the texture using functions such as Eq. (3). Because the 




Figure 7 Plot of hysteresis losses $(\mathrm{kG} \times \mathrm{Oe})$ vs $\Phi_{4}:$ a) $H_{\max }=3.0 \mathrm{Oe}$, b) $H_{\max }=5.0 \mathrm{Oe}$.

rearrangement of the main domain structure inside each crystallite is a major cause of the losses. The presented results (Figure 7) do not show any such correlation and are in contradiction with those published by Morris and Flowers. ${ }^{4}$ In highly oriented $\mathrm{Fe}-\mathrm{Si}$ steels, other texture related factors, such as the interaction between supplementary and main domain structure, may effect measured hysteresis loss data and improve the correlation.

\section{DISCUSSION}

It is difficult, without knowing the magnitude of all errors, to know what data points do not correlate with a certain function and whether one should increase the complexity of the function to try to account for any poor correlation. One further complication arises when the data obtained is a combination of an anisotropy related to the correlating function and one that is not. It is thus necessary to do a wide variety of test correlations before settling on a certain combination of correlating function and erroneous data points. For example, at lower field strengths, the relative permeability data consistently showed high values for $\beta=0$ and $10^{\circ}$ and low values for $\beta>60^{\circ}$ while at higher field strengths the data correlated well. The correlation at lower $H_{a}$ improved slightly if the basic fitting function, $\Phi_{4}$, was appended to with $\Phi_{6}$, but based on the success rate of the $\Phi_{4}$ correlation at the higher field strengths and the magntiude of the improvement in the fit attained when including $\Phi_{6}$, the $\beta=0$ an $10^{\circ}$ data was assessed to be anomalous. There are a number of possible explanations for this discrepancy in the data, the most likely being that due to the necessity of creating closure domain structures for domains not aligned with the direction of magnetization. Because of the strongly Goss nature of the texture existing in the samples used, large closure structures must exist for those domains oriented in the easy directions perpendicular to the rolling direction of the sheet. This means that, for magnetization in directions away from the rolling direction, some of the energy supplied by the magnetic field could go towards creating more complex 
Table 3 Fitting constants $A_{0}$, and $A_{4}$ for relative permeability measured at various places on the magnetization curves.

\begin{tabular}{rrrrrrrr}
\hline & $0.1 k G$ & $0.2 k G$ & $0.5 O e$ & $2.0 O e$ & $5.0 O e$ & $20.0 O e$ & $100.0 O e$ \\
\hline$A_{0}$ & 1587 & 2135 & 13,784 & 6435 & 2709 & 724 & 170 \\
$A_{4}$ & -9734 & $-13,486$ & $-96,475$ & -9449 & -3769 & -1053 & -185 \\
\hline
\end{tabular}

closure domain structures. Following this, as there is less need for creating closure domain structures for those domains oriented along the rolling direction of the sheet, magnetization in this direction could require less energy relative to magnetization in the other directions. It might be possible to explain the problems with the data for $\beta>60^{\circ}$ using the same hypothesis.

Ideally, the constants, $\left(A_{0}, A_{4}\right.$ and $\left.A_{6}\right)$ derived based upon the least squares fit of the anisotropy data should allow one to predict, given the first three non-zero coefficients of the ODF, the anisotropy of relative permeability, magnetic torque and magnetocrystalline energy for any similarly composed $\mathrm{Fe}-\mathrm{Si}$ steel of any texture, provided that such metallurgical factors as grain size and state of internal stress are similar. The constants $A_{4}$, and $A_{6}\left(A_{4}=(3.7 \pm 0.1) \times 10^{5} \mathrm{ergs} / \mathrm{cm}^{3}\right.$, $\left.A_{6}=(0.6 \pm 0.1) \times 10^{5} \mathrm{ergs} / \mathrm{cm}^{3}\right)$ calculated while correlating the anisotropy of magnetic torque with Eq. (3) provide good agreement with tabulated values for the $\mathrm{Fe}-\mathrm{Si}$, single crystal anisotropy constants for magnetocrystalline energy, $\left(K_{4}=3.5 \times 10^{5} \mathrm{ergs} / \mathrm{cm}^{3}, K_{6}=1.0 \times 10^{5} \mathrm{ergs} / \mathrm{cm}^{3}\right)$. Table 3 contains the $A_{0}$, and $A_{4}$ fitting constants for the correlation of the anisotropy of relative permeability of our Fe-Si sample with the texture. For our specimen, we did not obtain much correlation between the texture function and the anisotropy of hysteresis losses and further tests using specimens of varying textures are required. The authors at the time of this paper were unable to obtain such a variety of samples, and were therefore unable to rest the global nature of the derived constants $A_{0}$, and $A_{4}$. However, based on similar work done by Morris and Flowers, ${ }^{4}$ it is expected that the results would be good provided that the variation in the magnitude of any contaminating anisotropies is small; for weaker textures other possible anisotropies are less significant.

\section{CONCLUSIONS}

In this paper attempts were made to correlate the anisotropy of magnetic torque, relative permeability and hysteresis losses in a highly oriented $\mathrm{Fe}-\mathrm{Si}$ steel with the texture function.

$$
F(\alpha, \beta)=A_{0}+A_{4} \Phi_{4}(\alpha, \beta)+A_{6} \Phi_{6}(\alpha, \beta)
$$

This function was derived based upon an averaging, through the ODF, of the anisotropy of the single crystal properties and thus is not a suitable correlating function for those texture related anisotropies that exist only in the polycrystalline specimens. It was found that the anisotropy of magnetic torque, and thus of magnetocrystalline energy, and of relative permeability, were correlatable, and that, to within the error of the data, fits assuming $A_{6}=0$ produced sufficient 
accuracy. However, similar correlations with the anisotropy of hysteresis losses produced significantly poorer results.

\section{ACKNOWLEDGMENT}

One of the authors, P. Blandford would like to acknowledge that he is supported by a Canadian Steel Industry Research Association (CSIRA) scholarship.

\section{References}

1. Bunge, H. J. (1986). Experimental Techniques of Texture Analysis, p. 171, InformationgesellschaftVerlag.

2. Bunge, H. J. (1977). Texture of Crystalline Solids, 2, p. 169.

3. Bunge, H. J. (1982). Texture Analysis in Materials Science, p. 306, Butterworths \& Co., London.

4. Morris, P. R. and Flowers, J. W. (1981). Texture of Crystalline Solids, 4, 129-141.

5. Bunge, H. J. (1982). Texture Analysis in Materials Science, p. 308, Butterworths \& Co., London.

6. Szpunar, J. A., (ibid) Anisotropy of Magnetic Properties in Textured Materials.

7. Szpunar, J. and Ojanen, M. (1975). Metall. Trans. A 6a, pp. 561-567.

8. Brady, L. J. (1977). Journal of Materials Science 12, 2115-2123.

9. Sizoo, Z. (1929). Phys., 56, p. 649. 